> La libération efficace de médicaments dans l'organisme requiert l'usage de nanovecteurs non toxiques. Les matériaux actuels montrent des capacités de stockage faibles et des libérations trop rapides pour la plupart des principes actifs d'intérêt. Les nouveaux solides poreux hybrides, à cause de la diversité de leurs structures et de leurs porosités, présentent une alternative crédible à ce qui existe. La présente revue souligne les performances très attractives de carboxylates de fer(III) dans ce domaine pour encapsuler puis libérer de manière progressive des médicaments contre le cancer (antitumoraux) et le sida (antirétroviraux). Actifs en imagerie médicale, ils associent dans de bonnes conditions thérapie et diagnostic et ouvrent la voie à un traitement personnalisé des patients (la théragnostique). <

\section{Des nanovecteurs hybrides pour la restitution retard de médicaments antitumoraux et antiviraux}

Patricia Horcajada, Christian Serre, Gérard Férey, Patrick Couvreur, Ruxandra Gref

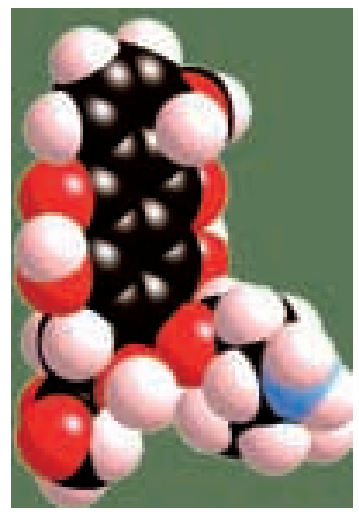

P. Horcajada, C. Serre, G. Férey : Institut Lavoisier (CNRS 8180), Université de VersaillesSaint-Quentin-en-yvelines, 45, avenue des États-Unis, 78035 Versailles, France. P. Couvreur, R. Gref : Faculté de pharmacie (CNRS 8612), Université Paris-Sud, 92296 Châtenay-Malabry, France. gferey@wanadoo.fr
Dans le domaine de la santé, un des grands défis actuels concerne la restitution efficace dans le corps humain de médicaments grâce à de nouveaux nanovecteurs non toxiques susceptibles d'un adressage ciblé dans l'organisme. Une thérapie efficace demande que ces vecteurs soient capables:

- de stocker des quantités importantes du médicament considéré ;

- d'éviter une restitution trop rapide (quelques minutes) dans l'organisme (ce que les Anglo-Saxons appellent le burst effect);

- de contrôler leur dégradation et de ne pas présenter d'effet toxique ;

- dêtre volontairement modifiables en surface pour contrôler les réactions in vivo;

- dêtre détectables par les techniques d'imagerie médicale.

\section{État de l'art}

Dans l'idéal, une fois toutes ces conditions remplies, ces nanovecteurs devraient permettre d'accéder à une nou- velle étape de la médecine moléculaire (la théragnostique) qui combine thérapie et diagnostic pour aboutir à un traitement réellement personnalisé des patients.

Pour un adressage efficace par voie intraveineuse durant tout son parcours jusqu'à la cible, il faut que le médicament (souvent instable, ou présentant une faible solubilité dans les milieux biologiques) puisse être protégé à l'intérieur de vésicules nanométriques (les vecteurs) qui assurent son intégrité et son transport jusqu'à l'organe, le tissu ou la cellule malades.

Les vecteurs actuellement utilisés [1-5] ne satisfont que très partiellement à ce cahier des charges. Ce sont le plus souvent (Figure 1):

- des liposomes, petites particules sphériques constituées d'une double couche de lipides (glycérophospholipides) servant d'enveloppe à différentes substances médicamenteuses. En fonction de leurs caractéristiques physico-chimiques, celles-ci peuvent être solubilisées soit dans la phase aqueuse interne des liposomes, soit dans les bicouches lipidiques. Ces sphères peuvent traverser les membranes des cellules et transporter les principes actifs jusqu'au cœur de celles-ci ;

- des micelles et des nanoémulsions, autres types de vésicules; 


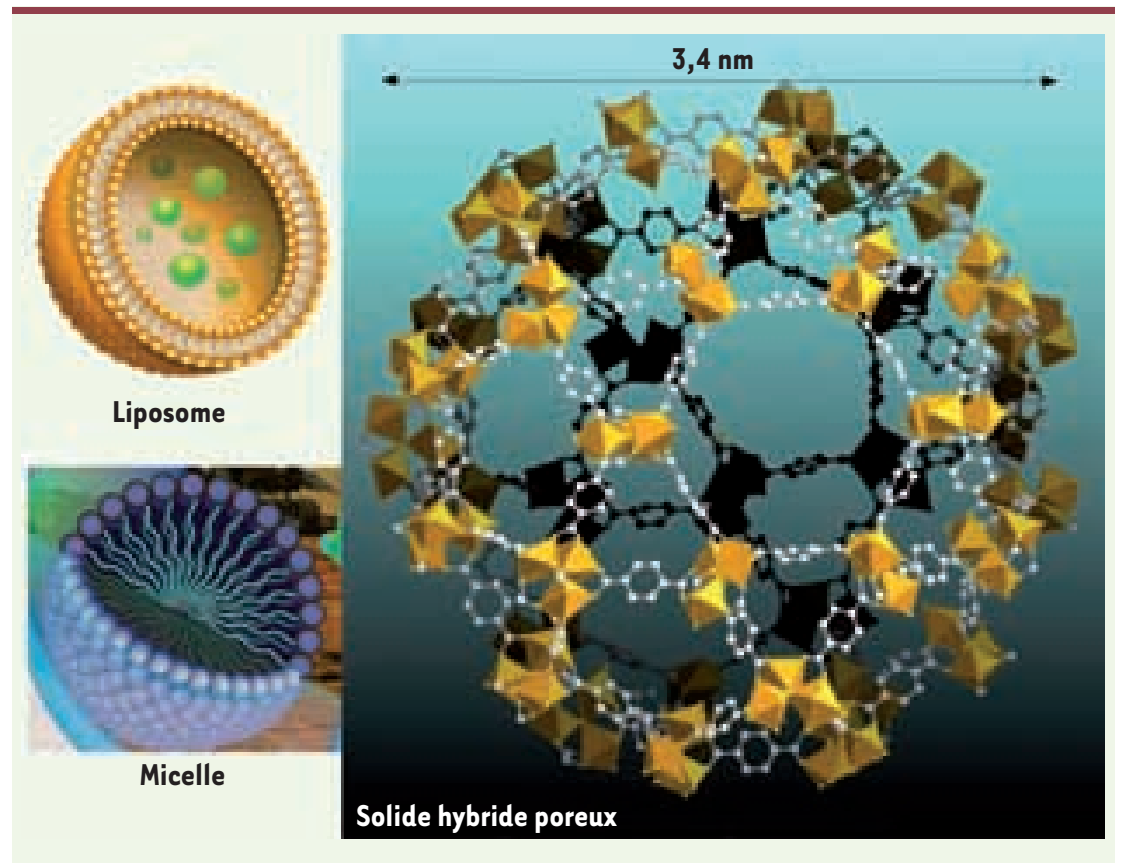

Figure 1. Aspect d'un liposome, d'une micelle et, par comparaison, d'un solide hybride poreux. S'agissant des liposomes et des micelles, les figures ne représentent qu'une seule vésicule, mais les agrégats réels ont des dimensions d'environ $100 \mathrm{~nm}$ pour les liposomes et de 30 à $50 \mathrm{~nm}$ pour les micelles.

squalénés obtenus sont beaucoup plus efficaces que les molécules d'origine dans des modèles de cancers expérimentaux (leucémies et tumeurs solides) car ils: (1) peuvent être administrés par voie intraveineuse ou orale; (2) favorisent la pénétration intracellulaire des analogues nucléosidiques; (3) ont un passage transmembranaire facilité ; et (4) sont protégés de la dégradation et du métabolisme.
- des nanoparticules, dans lesquelles le principe actif se trouve solubilisé, encapsulé ou adsorbé au sein de la matrice : organiques (typiquement polymériques: microcapsules, microsphères), inorganiques ou hybrides.

En particulier, les quantités de médicament transportées restent faibles (de l'ordre de 5 grammes de médicament pour 100 grammes de vecteur), limitées en cela par la faible taille des vésicules (quelques angströms ( $\left.1 \AA=10^{-10} \mathrm{~m}\right)$, assorties parfois du burst effect.

Nous venons de faire significativement progresser le domaine par deux avancées: la pégylation/squalénisation et l'utilisation de solides poreux hybrides comme vecteurs [31].

La pégylation a permis la conception de vecteurs dits « de deuxième génération » (nanoparticules, liposomes) capables d'atteindre les territoires pathologiques en évitant leur capture par le système réticuloendothélial (SRE). Le polyéthylène glycol (PEG) constitue un bouclier qui empêche l'adsorption des protéines sériques responsables de la reconnaissance par le SRE. Ce polymère très hydrophile améliore par ailleurs la compatibilité des biomatériaux. Les nanoparticules qui en sont recouvertes ont un temps de passage dans le torrent circulatoire très augmenté (quelques heures au lieu de quelques minutes) [6].

Dans le domaine du cancer, ces nouveaux vecteurs, qualifiés de «furtifs », ciblent le tissu tumoral avec une certaine spécificité par la combinaison de l'augmentation du temps de circulation des nanoparticules dans le sang et de la perméabilité vasculaire des vaisseaux sanguins irriguant la tumeur (effet $\varepsilon P R^{1}$ ).

Le concept de squalénisation tire profit de la conformation moléculaire compacte du squalène pour obtenir des systèmes nanoparticulaires en couplant cette molécule à des analogues nucléosidiques à activité anticancéreuse ou antivirale [7]. Les nanomédicaments

${ }^{1}$ L'effet EPR (pour Einstein Podolski Rosen) est un phénomène qui, en quantique, définit la non-séparabilité.

\section{Les nouveaux nanovecteurs hybrides poreux}

Ces solides ont une existence récente [8]. Ils sont obtenus par synthèse hydro-solvothermale $\left(150^{\circ} \mathrm{C}-30\right.$ bars $)$. Tridimensionnels, ils résultent de l'assemblage exclusivement par liaisons fortes d'agrégats inorganiques et de ligands organiques (essentiellement des carboxylates et des phosphonates) aisément modulables. Le nombre quasi infini de combinaisons entre parties organiques et inorganiques justifie l'actuel développement extensif de ces matériaux. Suivant la topologie de la structure qui en résulte, ces matériaux forment des pores soit rigides, soit hautement flexibles (variation de volume supérieure à $300 \%$ ) [9] sous l'action du médicament. Ces grands pores (jusqu'à 4,7 nm de diamètre), disposés régulièrement dans la structure, sont facilement modulables en fonction des applications choisies (Figure 1). De plus, suivant les cas, la paroi interne des pores peut être rendue hydrophile ou hydrophobe. Les structures flexibles autorisent une adaptation de la porosité à la forme de la molécule insérée. Maintenant connus sous le label MIL-n (MIL pour matériaux de l'Institut Lavoisier), nos produits correspondent aux deux cas. Certains font déjà l'objet d'une production industrielle (MIL-53 commercialisé par BASF sous le nom de Basolite-100). Dans l'étude présentée ici, sont utilisées deux structures flexibles (les téréphtalates MIL-53 et MIL-88) à base de tunnels de dimensions utiles 0,8-1,1 nm et deux structures rigides (carboxylates MIL-100 et -101 à très larges pores, de diamètre 2,5-3,4 nm), toutes à base de fer(III), cation choisi à la fois pour sa non-toxicité 


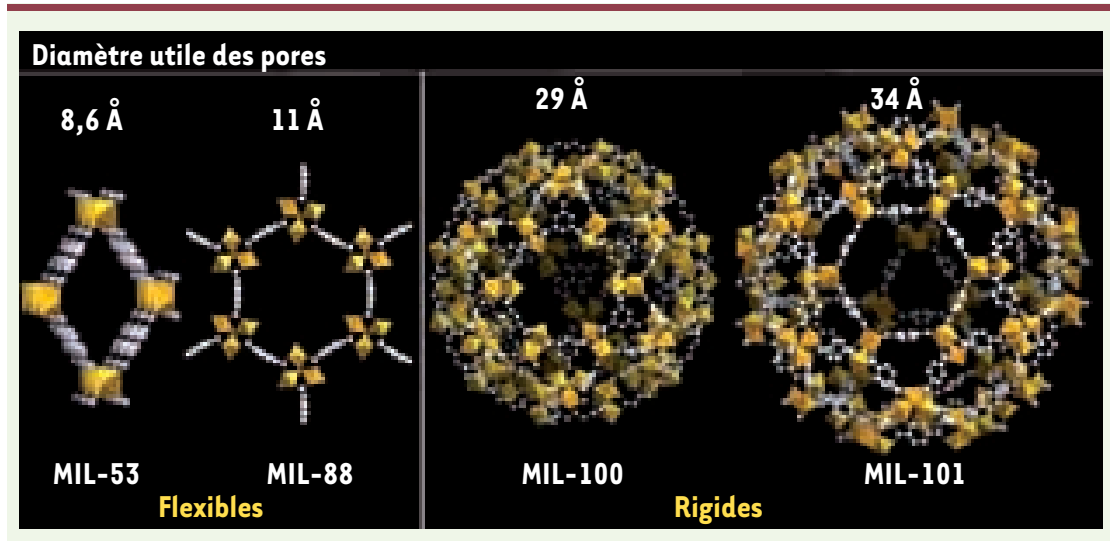

Figure 2. Structures, à la même échelle, des pores des nanovecteurs hybrides étudiés. Les parties inorganiques à base de fer(III) sont représentées sous forme d'octaèdres métalliques et les ligands par des assemblages de sphères. De gauche à droite: le MIL-53, un téréphtalate de fer dans lequel les octaèdres de fer forment des chaînes ; le MIL-88, autre téréphtalate de fer, lui aussi flexible, dans lequel la partie inorganique est constituée de trimères d'octaèdres, ce qui sera également le cas pour les deux phases suivantes; le MIL-100, trimésate de fer ; le MIL 101, téréphtalate de fer formant des mésopores.

et son activité en imagerie médicale (Figure 2). Ces phases autorisent déjà à $25^{\circ} \mathrm{C}$ le piégeage en grande quantité de gaz polluants, en particulier le $\mathrm{CO}_{2}$ et le méthane [10].

Avant d'étudier leurs possibilités pour le stockage et la restitution d'antitumoraux et d'antirétroviraux, nous avons validé notre méthode sur l'ibuprofène [11-14]. Qu'ils soient flexibles ou rigides, les MIL donnent des résultats spectaculaires, tant en termes de quantité stockée que de temps de libération (jusqu'à trois semaines pour le MIL-53). En particulier, le MIL-101 est capable de stocker 1,4 gramme d'ibuprofène par gramme de produit et de le libérer complètement en six jours.

\section{Nanofabrication et non-toxicité}

Deux étapes essentielles au respect du cahier des charges devaient être franchies en préalable à l'insertion des médicaments: (1) montrer que les solides choisis pouvaient être obtenus sous forme nanométrique afin d'autoriser ultérieurement des injections intraveineuses,

et (2) s'assurer de leur biodégradabilité et de leur nontoxicité intrinsèques.

En jouant sur tous les paramètres de la synthèse (nature du précurseur ferrique et du solvant - eau ou éthanol -, acidité de la solution, température), il a été à chaque fois possible (Figure 3) d'obtenir ces nanoparticules de dimensions compatibles $(<200 \mathrm{~nm})$ avec une injection sans dommages (Tableau I).

La présence de groupements réactifs en surface (sites métalliques, contre-ions ou groupements carboxyliques) permet de modifier celle-ci afin de contrôler le devenir in vivo des nanoparticules. Ainsi, le PEG leur confère une furtivité, mais des polysaccharides comme le dextrane et le chitosan ont aussi pu être associés, ouvrant d'autres perspectives comme le ciblage spécifique et la bioadhésion.

\begin{tabular}{|c|c|c|c|c|c|}
\hline Médicament & $\begin{array}{c}\text { Dimensions } \\
\text { (A) }\end{array}$ & $\begin{array}{l}\text { MIL-53 } \\
250 \mathrm{~nm} \text { * }\end{array}$ & $\begin{array}{l}\text { MIL-88 } \\
150 \mathrm{~nm} \text { * }\end{array}$ & $\begin{array}{l}\text { MIL- } 100 \\
200 \mathrm{~nm} \text { * }\end{array}$ & $\begin{array}{l}\text { MIL- } 101 \\
120 \mathrm{~nm} \text { * }\end{array}$ \\
\hline Amphiphile busulfan & $13,4 \times 3,5$ & & & & \\
\hline - Charge (\% en poids) & & 14,3 & 12 & 25,5 & nd \\
\hline - Efficacité (\% encapsulé) & & 17,9 & 6,3 & 31,9 & nd \\
\hline Hydrophile AZT-TP & $11,9 \times 9,1$ & & & & \\
\hline - Charge (\% en poids) & & 0,24 & 0,6 & 21,2 & 42 \\
\hline - Efficacité (\% encapsulé) & & 2,8 & 6,4 & 85,5 & 90,4 \\
\hline Hydrophile cidofovir & $10,8 \times 7,7$ & & & & \\
\hline - Charge (\% en poids) & & nd & 14 & 16,1 & 41,9 \\
\hline - Efficacité (\% encapsulé) & & nd & 81 & 46,2 & 68,1 \\
\hline Hydrophobe doxorubicine & $15,3 \times 11,9$ & & & & \\
\hline - Charge (\% en poids) & & nd & nd & 9,1 & nd \\
\hline - Efficacité (\% encapsulé) & & nd & nd & 11,2 & nd \\
\hline
\end{tabular}

Tableau I. Taille des nanoparticules, taille et performances des médicaments testés. Les dimensions en Å pour les molécules de médicaments sont celles estimées par simulation dans une atmosphère sous vide. La charge reflète la quantité de médicament encapsulée dans le solide, exprimée en \% du poids du médicament par rapport au poids du solide. L'efficacité est exprimée en \% de médicament encapsulé par le MIL par rapport à la quantité totale utilisée pour l'imprégnation (ou encapsulation). nd : non déterminé, l'encapsulation du médicament dans le MIL n'ayant pas été étudiée. *Taille des particules $(\mathrm{nm})$. 


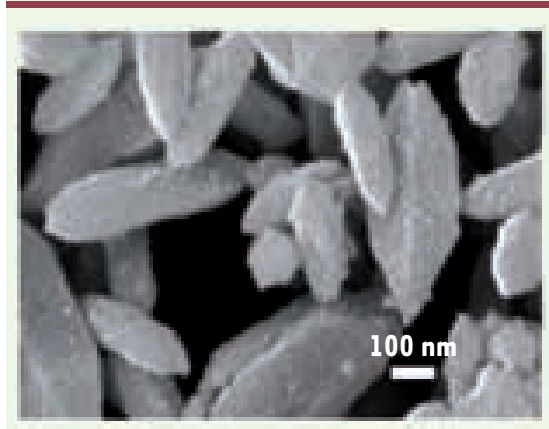

MIL-88A

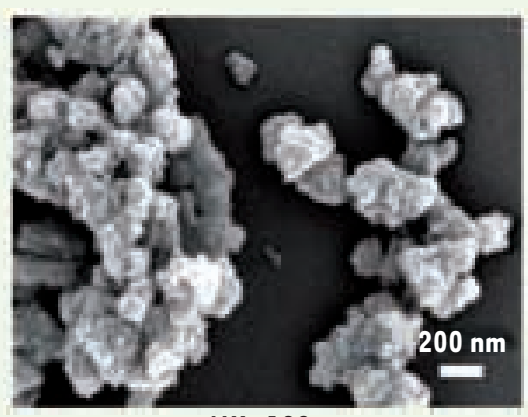

MIL-100

Figure 3. Micrographies de microscope électronique à balayage des nanoparticules de MIL-88 et de MIL-100.

ou leur libération trop rapide. Nous avons choisi quatre médicaments (Figure 4) aux caractéristiques différentes pour évaluer l'éventuelle généralité de notre méthode. Ces médicaments sont: l'agent antitumoral busulfan, les nucléotides cidofovir (CDV), forme monophosphorylée d'un

Le début de dégradation in vitro en conditions physiologiques n'intervient qu'après une semaine d'incubation à $37^{\circ} \mathrm{C}$, et cette dégradation est assortie d'une perte de cristallinité des particules et d'une libération significative des ligands constitutifs, notoirement non toxiques [15-18]. En termes de cytotoxicité, leur étude in vitro sur des macrophages de souris par la méthode $M T^{2}$ [19] démontre que la concentration pour laquelle les MIL sont non toxiques est comparable à celle des nanoparticules déjà existantes $(<60 \mathrm{mg} / \mathrm{ml})$ [20]. La toxicité in vivo a été testée après administration intraveineuse des nanoMIL à des rats Wistar femelles. Les doses injectées étaient très importantes $(220 \mathrm{mg} / \mathrm{kg}$ ). Par comparaison avec des groupes de rats contrôles, le comportement des animaux, le poids de leurs corps et organes ainsi que des paramètres sériques et hépatiques ne montrent aucune anomalie à l'issue des trois mois d'observation, ce qui prouve à la fois le caractère réversible de l'opération et la non-toxicité des nanoMIL. De plus, l'absence d'activation du cytochrome $P-450$ suggère l'excrétion directe des acides organiques. Enfin, une injection répétée pendant quatre jours de $150 \mathrm{mg} / \mathrm{kg} / \mathrm{jour}$ n'entraîne aucune manifestation d'effets toxiques au moins dans les dix jours suivants.

\section{Médicaments et performances d'encapsulation}

La non-toxicité avérée des nanoparticules de MIL autorise leur utilisation comme véhicules de médicaments anticancéreux et antirétroviraux dont l'efficacité est souvent limitée par leur forte insolubilité et/

${ }^{2}$ Le test MTT mesure la viabilité cellulaire et repose sur la mesure de la réduction, par les succinatedéshydrogénases mitochondriales, du MTT (bromure de 3-(4,5 diméthylthiazol-2-yl)-2,5 diphényl tétrazolium). phosphonate antiviral actif contre les cytomégalovirus, et l'azidothymine triphosphorylée (AZT-TP) utilisée contre le VIH (virus de l'immunodéficience humaine), tous deux hydrophiles, et, enfin, l'hydrophobe doxorubicine (doxo), un des agents les plus efficaces dans le cancer du sein.

Le busulfan, amphiphile antitumoral, est largement utilisé en combinaison avec des traitements de chimiothérapie lourde, en particulier dans les leucémies de l'enfant [21, 22]. Pris tel quel, il présente une faible stabilité en solution aqueuse et une importante toxicité hépatique due à sa microcristallisation dans le système microveineux du foie [23]. De plus, l'encapsulation actuelle de busulfan par les nanovecteurs disponibles, comme les liposomes ou les polymères, n'excède guère les $6 \%$ en poids [24], justifiant ainsi le test de nos nanoMIL comme une alternative intéressante à ce qui existe. En effet, l'imprégnation des MIL donne parfois des résultats exceptionnels. Par exemple, le MIL-100 stocke $25 \%$ en poids de busulfan, soit cinq fois plus que le meilleur polymère nanométrique et soixante fois plus que les liposomes ( $0,4 \%$ en poids) [23, 25]. Les MIL flexibles (MIL-53 et -88) ont des performances moindres, mais néanmoins supérieures à ce qui existe. Cela signifie que l'utilisation des nanoMIL représente un grand progrès dans la thérapie par le busulfan, en particulier parce qu'il faut moins de nanovecteurs pour administrer la dose requise de médicament, sans les risques

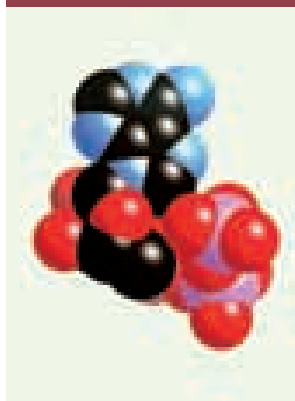

AZT-TP

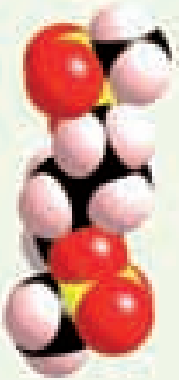

Busulfan

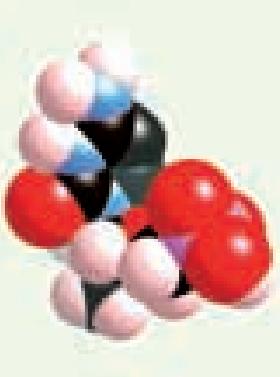

Cidofovir

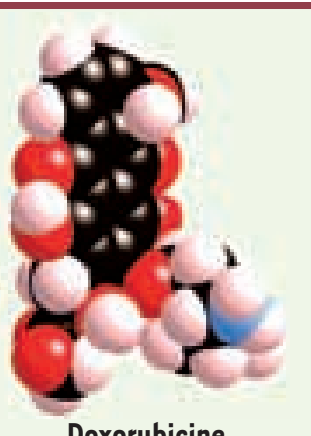

Doxorubicine
Figure 4. Structure des quatre médicaments encapsulés dans le carboxylate de fer. Les atomes sont indiqués par leur couleur: carbone (noir), oxygène (rouge), azote (bleu), soufre (jaune), phosphore (rose), hydrogène (blanc). 


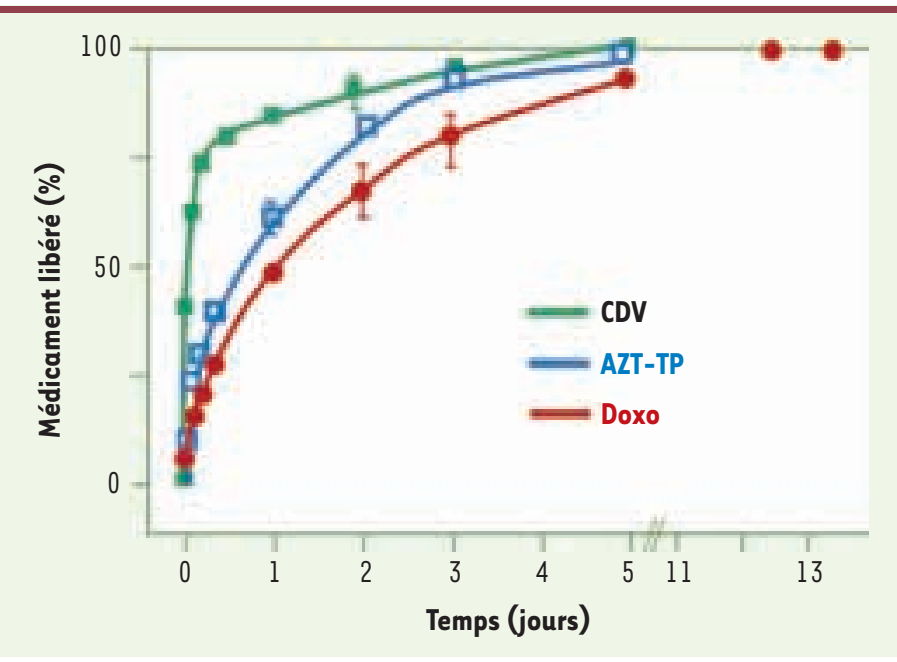

de recristallisation dans le système veineux hépatique. Des expériences in vitro montrent que le busulfan est libéré sous sa forme active, et son efficacité est constante, qu'il soit sous forme libre ou encapsulée.

Les nucléotides, peu stables en milieu biologique, ont de courtes durées de vie et entraînent parfois une résistance notable au médicament $[26,28]$. Le fort caractère hydrophile du CDV et de I'AZT-TP limite également leur pénétration intracellulaire, à cause de leur faible perméabilité membranaire [27, 28]. Bien que quelques nanovecteurs aient été mis au point pour pallier ces inconvénients, leur efficacité est très faible et s'accompagne de burst effects [29].

Tel n'est pas le cas pour les carboxylates de fer (Tableau I). Après imprégnation, ils présentent là encore des efficacités d'adsorption remarquables, parfois supérieures à $90 \%$. Par exemple, le MIL-100 stocke respectivement $25,21,16$ et $9 \%$ en poids de busulfan, AZT-TP, CDV et doxo. Le record appartient aux nanoparticules de MIL-101 qui encapsulent $42 \%$ d'AZT-TP et de CDV, valeur sans précédent comparée à celles reportées dans la littérature pour ces composés (1\%).

\section{Libération contrôlée}

Les libérations de I'AZT-TP, du CDV et de la doxo sont progressives, sans burst effect (Figure 5). La comparaison entre les cinétiques de libération et de dégradation des médicaments suggère que le processus de libération est gouverné, non pas par la dégradation des MIL, mais par leur diffusion à partir des pores. La libération totale de I'AZT-TP par le MIL-100 intervient ainsi en trois jours, alors que seuls $10 \%$ du vecteur se sont dégradés.

Ce résultat prometteur a incité à évaluer in vitro l'activité anti-VIH de I'AZT-TP, encapsulé dans le nanovecteur MIL 100 sur des cellules mononucléées de sang humain infectées par la souche VIH-l LAI. Pour une concentration de 200 nM d'AZT-TP encapsulée dans les nanoparticules du MIL-100, l'activité anti-VIH se traduit par $90 \%$ d'inhibition de la réplication du VIH comparativement à une inhibition nulle avec l'AZT libre.

\section{Utilité en imagerie}

Comme décrit précédemment, les structures MIL sont toutes à base de fer(III). Ce cation a été choisi en fonction de ses capacités à
Figure 5. Libération temporelle par les nanoparticules de MIL100. Du CDV (noir), de l'AZT-TP (bleu) et de la doxo (rouge) sous conditions physiologiques simulées (tampon PBS, $37^{\circ} \mathrm{C}$ ).

servir d'agent de contraste en imagerie médicale, de la même façon que le gadolinium [30] et le manganèse [8]. Après vérification que c'étaient bien les carboxylates (et non des hydroxydes issus de la dégradation) qui étaient responsables de l'effet, les mesures d'imagerie de résonance magnétique ont été faites sur des rats Wistar femelles après injection de suspensions de MIL-88 à 220 aux doses de 44 et $22 \mathrm{mg} / \mathrm{kg}$. Les séquences d'écho de gradient et d'écho de spin ${ }^{3}$ ont démontré que les images obtenues pour les organes traités sont plus sombres que celles issues d'organes d'animaux contrôles non traités (Figure 6), en particulier pour la rate et le foie. Trois mois après l'injection, l'identité des images d'organes d'animaux traités et contrôles a prouvé, a posteriori, que le séjour des nanoMIL dans ces organes était temporaire. L'efficacité des composés à base de fer tient à leur capacité à modifier, sous l'action d'un champ magnétique, les temps de relaxation des protons de l'eau dans le milieu.

\section{Conclusion}

Les faits prouvent que les carboxylates poreux de fer(III) représentent désormais les meilleurs nanovecteurs pour l'encapsulation et la libération de médicaments importants. Obtenus en solution soit aqueuse, soit alcoolique, au lieu d'utiliser des solvants organiques, ils fournissent le premier exemple de l'apport d'une technologie «verte» aux applications médicales. Ces nanovecteurs, non toxiques et biodégradables, agissent comme des éponges moléculaires capables de stocker, par simple imprégnation, des quantités très importantes de médicaments antitumoraux et antiviraux de polarités, de tailles et de fonctionnalités différentes, actives contre le cancer et le sida, avant leur libération contrôlée sans dommage sur plusieurs jours.

On peut considérer que ces nanovecteurs, qui respectent intégralement le cahier des charges, représentent le premier exemple de nanotransporteurs universels de médicaments et rendent possible un des rêves actuels de la médecine: disposer de nouveaux vecteurs qui conjuguent simultanément les possibilités de thérapie et de diagnostic. Cette nouvelle branche de la

3 E imagerie, il existe deux grandes familles de séquences, en fonction du type d'écho enregistré : les séquences d'écho de spin et les séquences d'écho de gradient. 


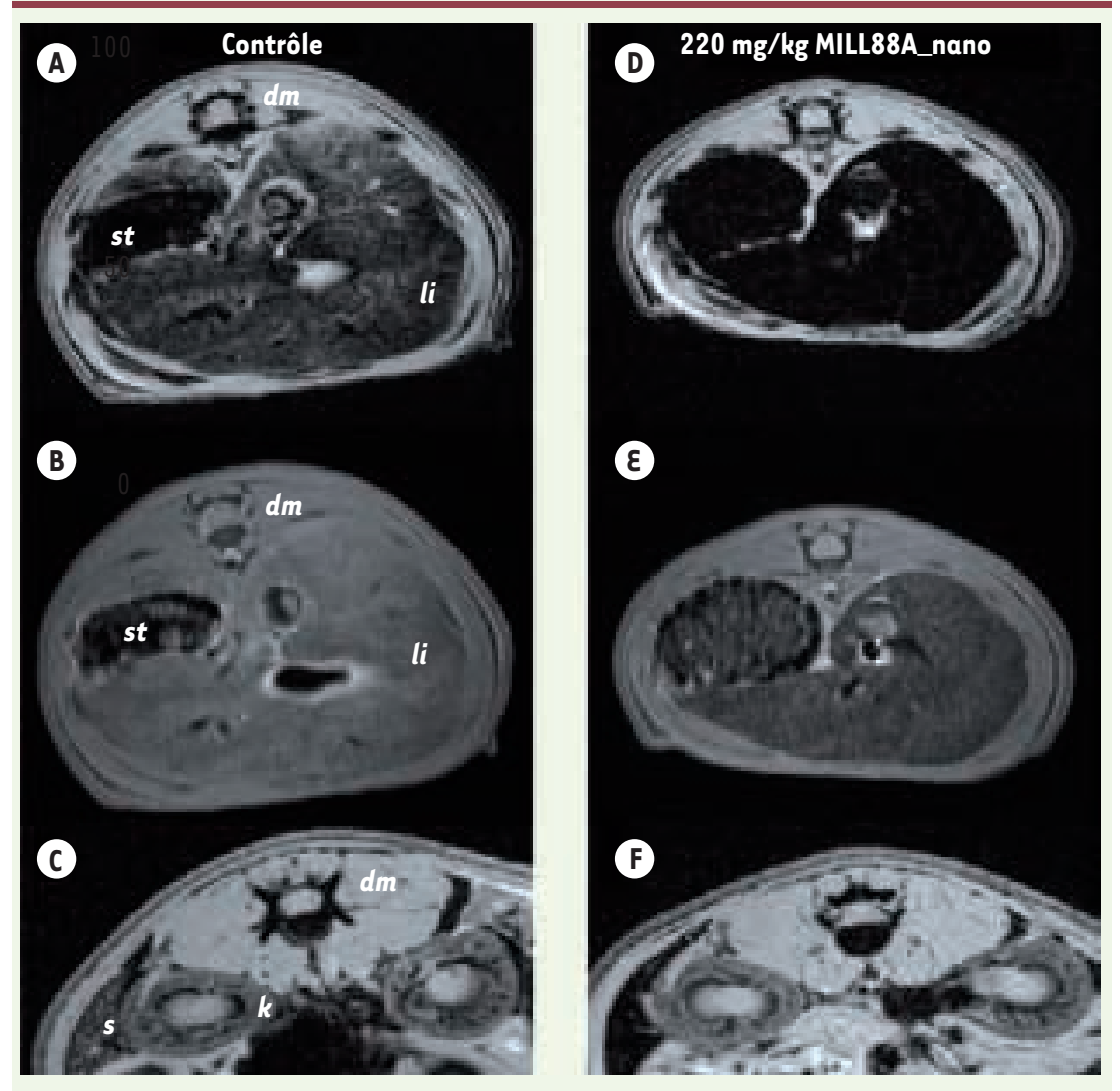

Figure 6. Images de résonance magnétique. Les images ont été acquises par des séquences d'écho de gradient ( $a, c, d, f$ ) ou d'écho de spin (b, e) sur des rats soit non traités ( $a$, $b, c)$ ou traités ( $d, e, f)$ par une injection de $220 \mathrm{mg} / \mathrm{kg}$ de MIL-88 visant le foie (a, b, d, e) et la rate (c, f). L'effet du produit est observable (organes plus sombres) $30 \mathrm{mn}$ après l'injection ( $\mathrm{dm}$ : muscle dorsal; $k$ : rein; $\mathrm{li}$ : foie; s: rate; st : estomac).

\section{SUMMARY}

Hybrid nanocarriers for controlled delivery of antitumour and retroviral drugs delivery

The efficient delivery of drugs in the body requires the use of non-toxic nanocarriers. Most of the existing materials show poor drug loading and/or rapid release of the proportion of the drug that is simply adsorbed (or anchored) at the external surface of the nanocarrier. The new porous hybrid solids, with the ability to tune their structures and porosities are well suited to serve as nanocarriers for médecine, la théragnostique, qui vise à un traitement personnalisé des patients, est appelée à un grand avenir dans les années qui viennent. Les MIL ouvrent une grande opportunité en ce sens.

\section{Et maintenant ?}

La voie est ouverte vers l'utilisation thérapeutique des nanovecteurs, mais est-elle perfectible? Sans aucun doute, et, maintenant que notre stratégie de caractérisation est bien établie, nos recherches actuelles visent la découverte de nouveaux matériaux, toujours aussi dépourvus de toxicité, à partir d'autres ions métalliques bénins pour l'organisme. Deux directions sont actuellement privilégiées: la première, au vu des performances exceptionnelles des MIL-100 et MIL-101, s'oriente vers la création rationnelle de matrices hybrides poreuses avec des cavités de plus en plus spacieuses pour accroitre les capacités de stockage, et donc diminuer la quantité de solide hybride injectée dans l'organisme sans affecter l'efficacité optimale du médicament. La seconde orientation, qui n'en est qu'à ses balbutiements, serait, au lieu d'encapsuler le médicament dans les pores, de se servir de lui comme ligand constitutif du squelette de l'hybride. Ainsi transporté, ce serait alors la dégradation du nanovecteur médicamenteux qui libérerait le principe actif, avec une cinétique qui serait fonction de la capacité du vecteur à s'autodétruire dans l'organisme. Des essais vont actuellement dans ce sens. Ils concernent également la modification superficielle de ce nanoMIL pour apporter aux vecteurs des propriétés de furtivité, de ciblage et de bioadhésion. $\diamond$ delivery and imaging applications. Here we show that specific non-toxic porous iron(III) - based metal - organic frameworks with engineered cores and surfaces, as well as imaging properties, function as superior nanocarriers for efficient controlled delivery of antitumour and retroviral drugs against cancer and AIDS. They also potentially associate therapeutics and diagnostics, and open the way for theranostics, or personalized patient treatments. $\diamond$

\section{CONFLIT D'INTÉRÊTS}

Les auteurs déclarent n'avoir aucun conflit d'intérêts concernant les données publiées dans cet article ni concernant les brevets.

\section{RÉFÉRENCES}

1. Peer D, Karp JM, Hong $S$, et al. Nanocarriers as an emerging platform for cancer therapy. Nat Nanotechnol 2007 ; 2 : 751-60.

2. Couvreur P, Gref R, Andrieux K, Malvy C. Nanotechnology for drug delivery: Applications to cancer and autoimmune diseases. Prog Solid State Chem 2006 ; 34 : 231-5.

3. Gref R, Minamitake Y, Peracchia MT, et al. Biodegradable long-circulating polymeric nanospheres. Science $1994 ; 263$ : 1600-3.

4. Gabizon A. Stealth liposomes and tumor targeting: One step further in the quest for the magic bullet. Clin Cancer Res $2001 ; 7: 223-5$.

5. Hasan AS, Socha M, Lamprecht A, et al. Effect of the microencapsulation of nanoparticles on the reduction of burst release. Int J Pharm 2007; $344: 53-61$.

6. Gref R, Minamitake $Y$, Peracchia MT, et al. Biodegradable long-circulating polymeric nanospheres. Science $1994 ; 263: 1600-3$. 
7. Couvreur P, Stella B, Reddy LH, et al. Squalenoyl nanomedicines as potential therapeutics Nano Lett $2006 ; 6: 2544-8$.

8. Férey G. Hybrid porous solids: past, present, future. Chem Soc Rev 2008 ; 37 : 191-241.

9. Férey G, Serre C. Large breathing effects in three-dimensional porous hybrid matter: facts, analyses, rules and consequences. Chem Soc Rev $2009 ; 38: 1380-99$.

10. Llewellyn PL, Bourrelly $\mathrm{S}$, Serre $\mathrm{C}$, et al. High uptakes of $\mathrm{CO}_{2}$ and $\mathrm{CH}_{4}$ in mesoporous metal organic frameworks MIL-100 and MIL-101. Langmuir 2008; $24: 7245-50$.

11. Horcajada P, Serre C, Vallet-Regí M, et al. Metal_organic frameworks as efficient materials for drug delivery. Angew Chem Int $\varepsilon d$ Engl $2006 ; 45: 5974-8$.

12. Horcajada P, Serre C, Maurin G, et al. Flexible porous metal organic frameworks for a controlled drug delivery. J Am Chem Soc 2008 ; $130: 6774-80$.

13. Horcajada P, Serre C, Gref R, et al. Nanoparticules hybrides organiques inorganiques à base de carboxylates de fer. PCT applications PCT/FR2008/001366, ler $^{\text {er }}$ octobre 2008.

14. Horcajada P, Serre C, Gref R, et al. Solides hybrides organique-inorganique à surface modifiée. PCT applications PCT/FR2008/001367, $1^{\text {er }}$ octobre 2008

15. Sheftel V0. Indirect food additives and polymers: migration and toxicology. Boca Raton Lewis Publishers, 2000 : 148-54.

16. http://www.chem.unep.ch/irptc/sids/0ECDSIDS/100-21-0.pdf> (2008).

17. http://www.chemicalland21.com/specialtychem/perchem/TRIMESIC \%20ACID.htm> (2008).

18. http://www.sciencelab.com/xMSDS-Fumaric_acid-9927173> (2008).

19. Mosmann T. Rapid colorimetric assay for cellular growth and survival: application to proliferation and cytotoxicity assays. J Immunol Methods 1983 ; 65 : 55-63.

20. Vauthier C, Dubernet C, Chauvierre C, et al. Drug delivery to resistant tumors: the potential of poly (alkyl cyanoacrylate) nanoparticles. J Control Release 2003 ; 93 : 151-60.

21. Vassal G, Deroussent A, Challine D, et al. Is $600 \mathrm{mg} \cdot \mathrm{m}^{-2}$ the appropriate dosage of busulfan in children undergoing bone marrow transplantation? Blood $1992 ; 79: 2475-9$.

22. Vassal G, Gouyette A, Hartmann 0, et al. Pharmacokinetics of high-dose busulfan in children. Cancer Chemother Pharmacol $1989 ; 24: 386-90$.
23. Slattery JT, Sanders JE, Buckner CD, et al. Graft-rejection and toxicity following bone marrow transplantation in relation to busulfan pharmacokinetics. Bone Marrow Transplant 1995 ; 16 : 31-42.

24. Layre A, Gref R, Richard J, et al. Nanoparticules polymériques composites. FR 04 07569, 7 juillet 2004.

25. Hassan Z, Nilsson C, Hassan M. Liposomal busulphan: bioavailability and effect on bone marrow in mice. Bone Marrow Transplant 1998 ; 22 : 913-8.

26. Thierry AR, Vigé D, Coughlin SS, et al. Modulation of doxorubicin resistance in multidrug-resistant cells by liposomes. FASEB J 1993 ; $7: 572-9$.

27. Loke SL, Stein CA, Zhang XH, et al. Characterization of oligonucleotide transport into living cells. Proc Natl Acad Sci USA 1989; 86 : 3474-8.

28. Kukhanova M, Krayevsky A, Prusoff W, Cheng YC. Design of anti-HIV compounds: From nucleoside to nucleoside 50-triphosphate analogs. Problems and perspectives. Curr Pharm Des $2000 ; 6: 585-98$.

29. Hillaireau H, Le Doan T, Besnard M, et al. Encapsulation of antiviral nucleotide analogues azidothymidine-triphosphate and cidofovir in poly(isobutyl cyanoacrylate) nanocapsules. Int J Pharm 2006 ; $324: 37-42$.

30. Taylor KM, Jin A, Lin W. Surfactant-assisted synthesis of nanoscale gadolinium metal_organic-framework for potential multimodal imaging. Angew Chem Int Ed Engl $2008 ; 47: 7722-5$.

31. Horcajada P, Chalati T, Serre C, et al. Porous metal-organic framework nanoscale carriers as a potential platform for drug delivery and imaging. Nat Mater $2010 ; 9: 172-8$

\section{TIRÉS À PART}

G. Férey

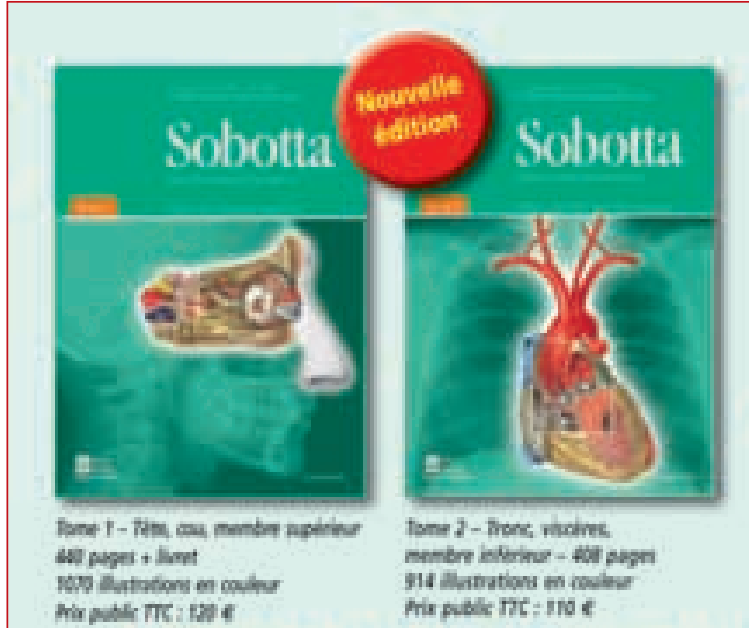

SOBOTTA - Atlas d'anatomie humaine en 2 tomes

R. PUTZ, R. PABST, coordonnateurs

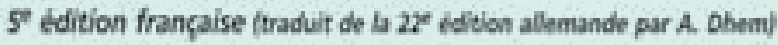

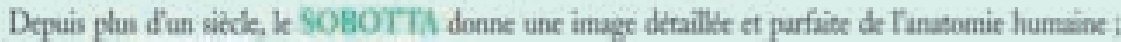

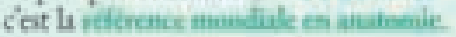

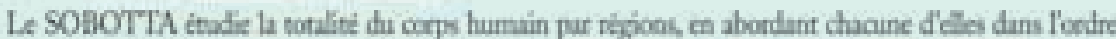

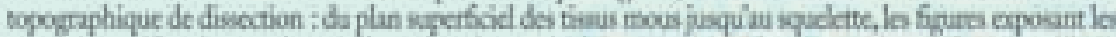

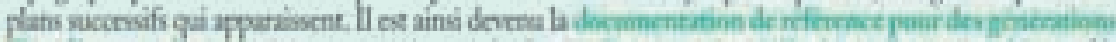

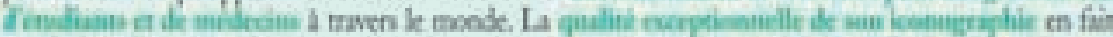

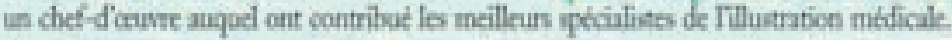

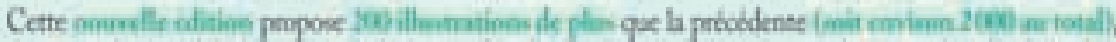
ance une intetgration importante des procédts dimigoric. De phus, de nombetuses dés doricntation

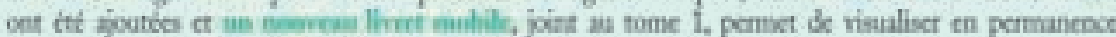

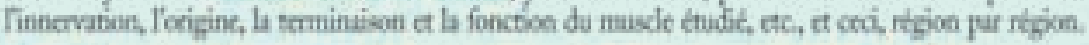

\section{Ce véritable chef-d'cuvre est L.A rêférence fiable pour votre pratique quotidienne.}

En vente chez votre Iibraire spécialise, par correspondance ou sur notre site wwweminter.fr

\section{$\cdots 8<$ \\ Bon de commande à retourner complete a

\begin{tabular}{|c|c|}
\hline & Quantite \\
\hline 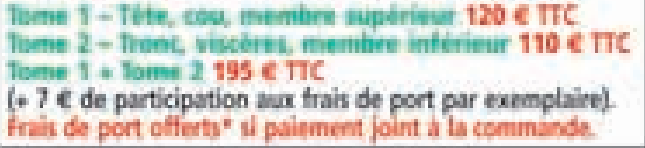 & \\
\hline
\end{tabular}

[*France metropolitaine, Suisse, UE. Autres, nous conuiten]

Adresse:

Code postal : .......... vile :

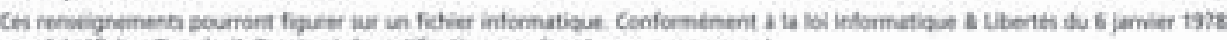

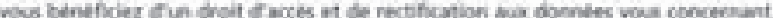

\section{M inter - Allee de la croix Bossée - 94234 Cachan Cedex}

le joins mon réglement a la commande :

Montant total de 2 C Cheque bancaire ou postal payable en France

a Yordre de : EMINTER (Une facture acquittice sera jointe au colis)

Carte bancaire $n^{*}$

Date drexpiration :

Les 3 demiers chiffres situes au dos de votre carte bancaire 